\title{
REVISION
}

Perfil de seguridad a corto plazo de zolpidem: medidas objetivas de efectos cognitivos.

A. C. DeClerk, J. C. Bisserbe...

ARTICULOS ORIGINALES

Fiabilidad interevaluador de la $\mathrm{CIE}-10$ en pacientes chinos.

A. H. T. Pang, G. S. Ungvari, C. K. Wong, T. Leung.

Un estudio clínico de la fluoxetina en depresivos con retardo/embotamiento afectivo frente a depresivos agitados/ansiosos: ¿existen efectos diferenciales?

A. Partiot, R. Jouvent, A. Pierson, P. Baruch, J. N. Beuzen, S. Ammar, D. Widlöcher..

Niveles de globulina fijadora de cortisol en el trastorno bipolar.

E. Vieta, C. Gastó, M. J. Martínez de Osaba, A. Otero, E. Nieto, L. Pintor, J. Blanch, J. Vallejo....

La respuesta inicial al fármaco activo y al placebo predice la evolución del tratamiento antidepresivo.

S. Priebe, M. Bröker

Estilo de atribución e ideas delirantes: una investigación basada en el contenido delirante.

H. M. Sharp, C. F. Fear, D. Healy......

\section{COMUNICACION BREVE}

Ataques de pánico en el sueño en pacientes con trastorno de pánico: la asociación con depresión mayor.

M. Y. Agargün, H. Kara

NOTICIAS DE LA AEP

El lanzamiento europeo de la psicoterapia interpersonal en el X Congreso Mundial de Psiquiatría.

J. Solé-Puig 


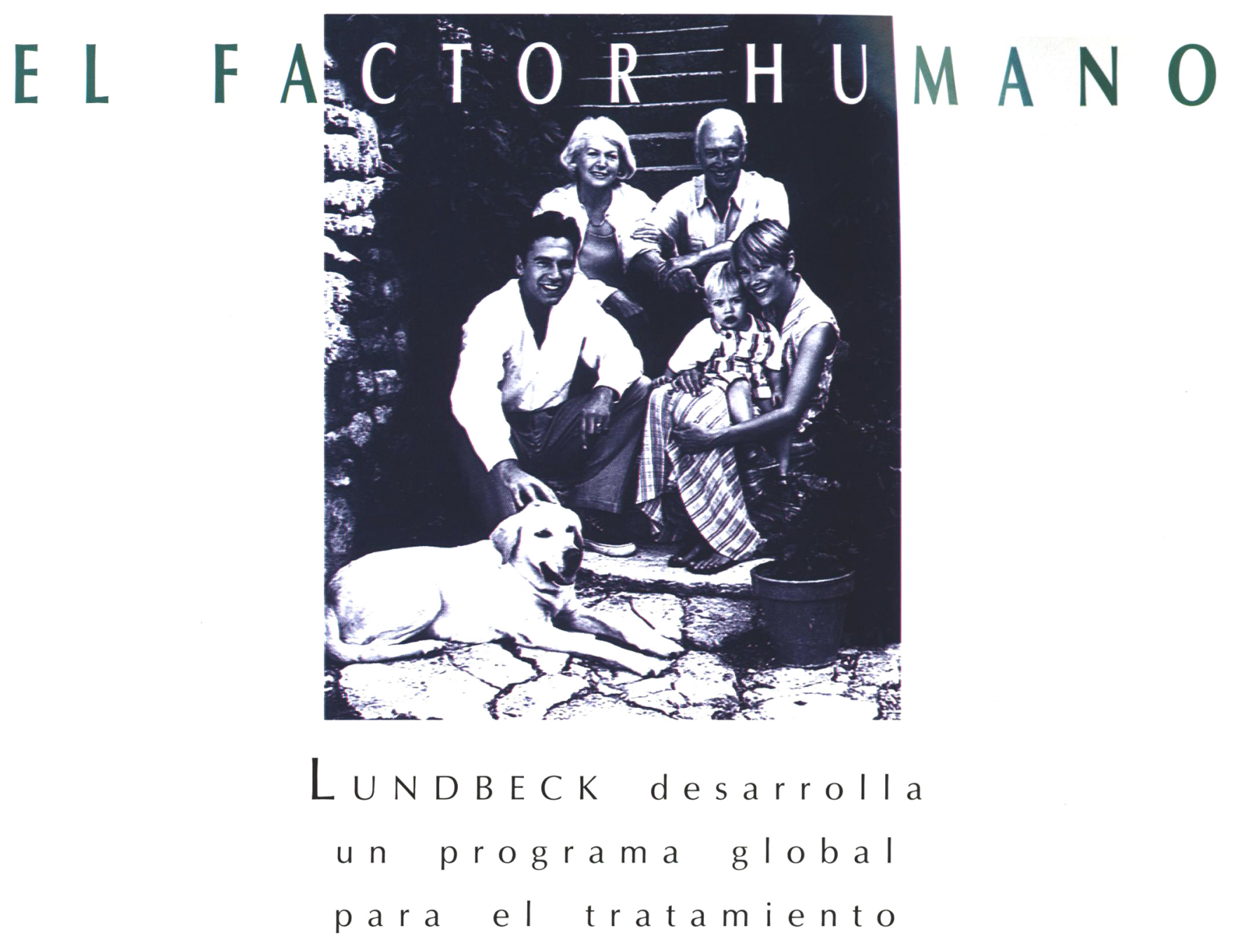

más hum a no y científico

de la depresión y la esquizofrenia

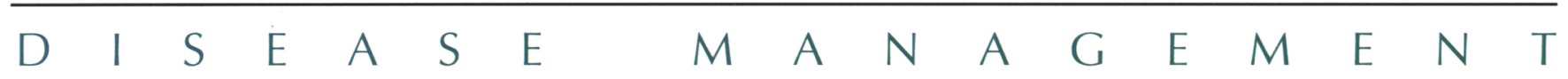
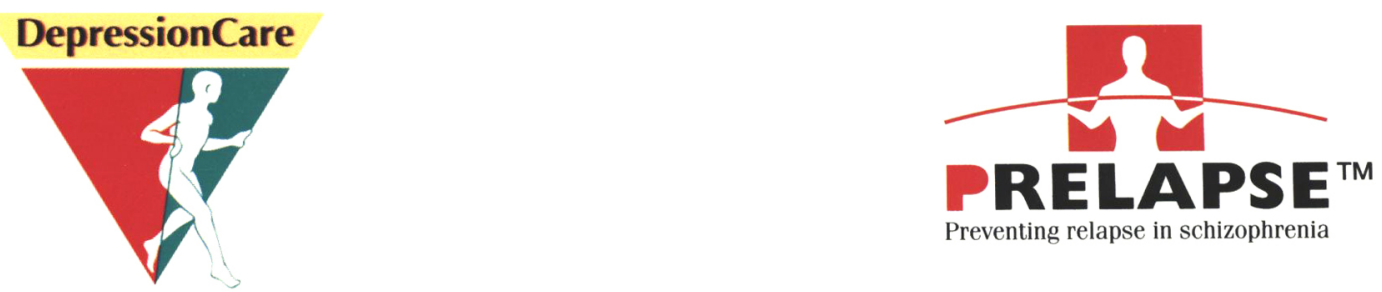

Nuestros esfuerzos se dirigen a profesionales de la salud mental, pacientes y familiares con el objetivo prioritario de recoger, compartir y utilizar la información para mejorar la calidad de vida del enfermo crónico

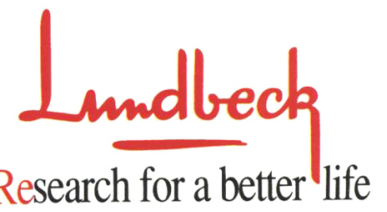




\section{EUROPEAN PSYCHIATRY}

Editors-in-chief: P Boyer, Y. Lecrubier (Paris).

Secretariat: Hôpital de la Salpêtrière, INSERM U 302, Pavillon Clérambault, 47, bd de l'Hôpital, 75651 Paris cedex 13, France. Tel: (33) 01421616 56. Fax: (33) 0145852800

Editors: JD Guelfi (Paris/Villejuif), R. Murray (London), M Maj (Naples), CB Pull (Luxembourg), M. Ackenheil (Munich), P Bech (Copenhagen) Editors Emeritus: C Ballús (Barcelona), H Heimann (Tübingen)

\section{Consejo Editorial}

J Adès, Colombes, France

HS Akiskal, Rockville, MD, USA

NC Andreasen, Iowa City, IA, USA

J Angst, Zurich, Switzerland

M. Ansseau, Liège, Belgium

P Baumann, Lausanne, Switzerland

H Beckmann, Wïrzburg, Germany

P Berner, Vienna, Austria

A Bertelsen, Risskov, Denmark

J Bibert, Cadiz, Spain

J Birley, London, UK

JC Bisserbe, Meudon, France

B Bondy, Munich, Germany

JP Boulenger, Sherbrooke, Canada

M Bourgeois, Bordeaux, France

F Brambilla, Milan, Italy

I Brockington, Birmingham, UK

A Clare, Dublin, Ireland

F Clerget-Darpoux, Paris, France

V Conde Lopez, Valladolid, Spain

S Consoli, Paris, France

$\mathrm{P}$ Cosyns, Antwerp, Belgium

J Cottraux, Lyon France

$\mathrm{M}$ von Cranach, Kaufbeuren, Germany

A Dahl, Oslo, Norway

JM Danion, Strasbourg, France

JFW Deakin, Manchester, UK

$\mathrm{M}$ de Bonis, Le Kremlin Bicêtre, Paris, France

H Dufour, Lausanne, Switzerland

R Engel, Munich, Germany

T Fahy, Galway, Ireland

L Farde, Stockholm, Sweden

A Féline, Le Kremlin Bicêtre, Paris, France

A Fernandes da Fonseca, Porto, Portugal

F Ferrero, Geneva, Switzerland
M Fichter, Prien am Chiemsee, Germany

H Freeman, London, UK

HJ Gaertner, Tübingen, Germany

D Goldberg, Manchester, UK

I Hand, Hamburg, Germany

H Häfner, Mannheim, Germany

T Helgason, Reykjavik, lceland

H Hippius, Munich, Germany

A Jablenski, Sofia, Bulgaria

E Johnston, Edinburgh, UK

S Kasper, Vienna, Austria

M Kastrup, Hvidovre, Denmark

D Kemali, Naples, Italy

R Kendell, Edinburgh, UK

D Klein, New York, NY, USA

R Klein, New York, NY, USA

$S$ Langer, Paris, France

J Lellouch, Villejuif, France

$P$ Lemoine, Lyon, France

T Lemperière, Colombes, France

JP Lépine, Paris, France

OM Lesch, Vienna, Austria

SW Lewis, London, UK

H Lôo, París, France

JJ López-Ibor, Madrid, Spain

P McGuffin, Cardiff, $U K$

W Maier, Mainz, Germany

A Mann, London, $U K$

K Mann, Tübingen, Germany

I Marks, London, $U K$

J Marlet, Venray, The Netherlands

J Massanna, Barcelona, Spain

J Mendlewicz, Brussels, Belgium

HJ Möller, Munich, Germany

N Müiller, Munich, Germany
M Musalek, Vienna, Austria

D Naber, Munich, Germany

E O'Callaghan, Dublin, Ireland

Y Ono, Tokyo, Japan

M Patris, Strasbourg, France

J Pellet, Saint-Etienne, France

C Perris, Umeä, Sweden

P Pichot, Paris, France

T Pohlmächer, Munich, Germany

H Pope, Belmont, MA, USA

AJ Puech, Paris, France

G Racagni, Milan, Italy

N Retterstøl, Oslo, Norway

$\mathrm{M}$ A Ron, London, $U K$

R Rosenberg, Risskov, Denmark

M Roth, Cambridge, UK

F Rouillon, Colombes, France

J Saiz-Ruiz, Madrid, Spain

A Sánchez-Blanque, Zaragoza, Spain

N Sartorius, Geneva, Switzerland

F Schulsinger, Copenhagen, Denmark

G Sedvall, Stockholm, Sweden

L Singer, Strasbourg, France

CN Stefanis, Athens, Greece

E Straube, Tübingen, Germany

E Taylor, London, UK

P Taylor, London, $U K$

L Träskman-Bendz, Lund, Sweden

$\mathrm{J}$ Vallejo, Barcelona, spain

L Waintraub, Paris, France

D Widlöcher, Paris, France

J Wilmotte, Charleroi, Belgium

J Wing, London, UK

FT Zimmer, Tübingen, Germany

$\mathrm{J}$ Zohar, Beer-Sheva, Israel

\section{Asociación de Psiquiatría Europea}

President: J Angst (Zürich); Past President: R Murray (London); President Elect: N Sartorius (Geneva); Secretary General: CB Pull (Luxembourg); Treasurer: M Patris (Strasbourg); Counsellors: H Häfner (Mannheim), Y Lecrubier (Paris); Section: L Singer (Strasbourg).

European Psychiatry, edición original, es publicada por Editions Scientifiques Elsevier, 141, rue de Javel, 75747 París, Francia. Indexada en: Biological Abstracts/Biosis, CNRS/Pscal, Current Contents/Clinical Medicine and Social Behavioral Sciences, Excerpta Medica/EMbase, Psychological Abstracts

Director de la Edición Española: C. Ballús

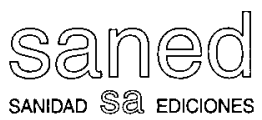

Sanidad y Ediciones (SANED, S.A.). C/ Paseo de la Habana, 202 bis. 28036 MADRID. Telf.: (91) 3594092

Caspe, 172, 4. ${ }^{\circ}$-A. BARCELONA. Telf.: (93) 2472411.

Directora Editorial: Alicia Martínez Magro.

Suscripciones: SANED, S.A. Paseo de la Habana, 202 bis. 28036 MADRID.

Publicación Bimensual ( 8 números al año).

Composición: Artecomp, S.L. Albarracín, 50-1.․ Madrid. Imprime: D.G.B. Gonzalo Dávila, 22. Madrid.

Soporte válido M. Sanidad: SV 93040 R. ISSN: 1134-0665. Depósito Legal: M-5991-1994.

Copyright de los textos originales 1997 . Reservados todos los derechos. Ninguna parte de esta publicación puede ser reproducida, transmitida

en ninguna forma o medio alguno, electrónico o mecánico, incluyendo fotocopias, grabaciones o cualquier sistema de recuperación de almacenaje de información, sin la autorización por escrito de los titulares del Copyright. 


\section{EUROPEAN PSYCHIATRY. EDICION ESPAÑOLA}

\author{
Volumen 4, Número 6, Septiembre 1997
}

\section{REVISION}

Perfil de seguridad a corto plazo de zolpidem: medidas objetivas de efectos cognitivos.

A. C. DeClerk, J. C. Bisserbe.

\section{ARTICULOS ORIGINALES}

Fiabilidad interevaluador de la CIE-10 en pacientes chinos. A. H. T. Pang, G. S. Ungvari, C. K. Wong, T. Leung.

Un estudio clínico de la fluoxetina en depresivos con retardo/embotamiento afectivo frente a depresivos agitados/ansíosos: ¿existen efectos diferenciales?

A. Partiot, R. Jouvent, A. Pierson, P. Baruch, J. N. Beuzen, S. Ammar, D. Widlöcher.

Niveles de globulina fijadora de cortisol en el trastorno bipolar.

E. Vieta, C. Gastó, M. J. Martínez de Osaba, A. Otero, E. Nieto, L. Pintor, J. Blanch, J. Vallejo

La respuesta inicial al fármaco activo y al placebo predice la evolución del tratamiento antidepresivo.

S. Priebe, M. Bröker .

Estilo de atribución e ideas delirantes: un investigación basada en el contenido delirante.

H. M. Sharp, C. F. Fear, D. Healy

\section{COMUNICACION BREVE}

Ataques de pánico en el sueño en pacientes con trastorno de pánico: la asociación con depresión mayor.

M. Y. Agargün, H. Kara

\section{NOTICIAS DE LA AEP}

El lanzamiento europeo de la psicoterapia interpersonal en el X Congreso Mundial de Psiquiatría.
FICHA TECNICA BESITRANQ(Sertralina).Composición: Cada comprimido contiene como principio
activo:BESTTRANQ 50 mg: Sertralina (D.C.I) (Clorhidrato), $50 \mathrm{mg}$; BESTRANQ $100 \mathrm{mg}$. Sertralina (D.C.1) (Clornidrato), $100 \mathrm{mg}$. BESITRAN(i) (molécula investigada y desarirolidada portraliza (D.C...) (Clorhidrato), por Pfizer) es un inhibidor especifico de la recaptación neuronal in vitro $€$ in vivo de seroTras una respuesta satisfactoria, la continuación del tratamiento con Sertralina es eficaz para prevenir las recaidas del episodio inicial de depresión o la recurrencia de futuros eppipara preventir las recaidas del episodio inicial de depresión o la recurrencia de futuros epp.
sodios depresivos. Tratamiento de los trastornas obsesivo-compulsivos. Posologia y forma sodios depresivos. Tratamiento de los trastornos obsesivo-compu'sivos. Posologia y forma de administracion: BESITRAN (Sertralinal debe administrarse por via oral en una sola dosis diaria por la mańana o por la noche. La dosis terapéutica es 50 mgldia. En casa de no $200 \mathrm{mg} / \mathrm{d}$ í. La aparición del efecto terapéutico puede observarse en 7 dias, aunque normalmente se necesitan de dos a cuatro semanas para obtener una actividad antidepresiva completa. Durante los períodos prolongados de mantenimiento del tratamiento, la dosis debe ajustarse al nivei mínimo eficaz en función de la respuesta terapéutica. Uso en niños y ancianos: Puede utilizarse el mismo rango de dosis en pacientes jóvenes y en ancianos No se ha establecido la seguridad y eficacia de Sertralina en niños. Contraindicaciones Hipersensibilidad conocida a Sertralina. Advertencias / precauciones IEnfermedad Cardiaca: Sertralina no produce cambios clinicamente significativos de la presión arterial frecuencia cardiaca ni ECG. No obstante, es necesario tomar las precauciones habituales en los pacientes con cardiopatias. Epilepsia: Puesto que Sertralina no ha sido evaluada en pacientes con un trastorno convulsivo, debe evitarse la administración de Sertralina en pacientes con epilepsia inestable, y los pacientes con epilepsia controlada deben ser cuidadosamente supervisados. El fármaco debe interrumpirse en cualquier paciente que desarrolle convulsiones. Insuficienca Hepática: El uso de Sertralina en estos pacientes debe rearrolle convulsiones. Insuficienca Hepática: El uso de Sertralina en estos pacientes debe realizarse con precaución, considerando una posible redución de la dosis o de la frecuencia do administraciónl. Insuficiencia Renal: En esta población de pacientes no se han estudiado do forma adecuada los parámetros farmacocinéticos de Sertralina en estado estable, por li que se recomienda precaucion cuando Sertralina se administre a pacientes con insuficiencia renal. Ancianos: El perfil y la incidencia de reacciones adversas en el anciano fueron similares al de los pacientes más jovenes. IMAOs: No debe administrarse Sertralina en asociación con un MAO, y deben transcurrir 14 dias entre la interrupción del tratamiento con los IMAOs y el inicio del tratamiento con BESITRAN (Sertralinal. A su vez no deben administrarse IMAOS antes de que hayan transcurrido 2 semanas, una vez suspendido el tsatamiento con Sertralina. Activación de Maniałtipomania: Se han comunicado casos de manía e hipomania en aproximadamente el 0,4\% de los pacientes tratados con Sertralina. Suicidio: Puesto que la posibilidad de un intento de suicidio es inherente a la enfermedad depresiva grave y puede persistir hasta que aparece una remisión significativa, los pacientes deben ser controlados estrechamente al principio del tratamiento. Embarazo y lactancia: BESTTRAN8 (Sertralina) no debe utilizarse durante el embarazo o la lactancia, a menos que los beneficios superen los posibles riesgos. Las mujeres que potencialmente puedan quedar embarazadas, deben emplear un método anticonceptivo, si están recibiendo BESSTRANQ ( Sertralina), Efectos sobre la capacidad para conducir vehículos y utilizar maquinaria: Sertralina no ejerce efectos sobre la función psicomotora. Sin embargo, como los antidepresivos pueden alterar la capacidad fisica o mental requerida para el desempeño de tareas potencialmente peligrosas, como la conducción de vehiculos o el uso de maquing ria, el paciente debe ser advertido al respecto. Interacciones: IMAOs: Ner Advertencies Precauciones). Alimentos: Los alimentos no modifican significativamente la biodisponibiliPrecauciones. Al Alcolo dad de Sertralina. Alcohol: La admininistración conjunta de Sertralina y alcohol no potencio los efectos del alcohol sobre las funciones cognitiva y psicomotora en sujetos sanos;'s sin embargo, no se recomienda el uso concomitante de Sertralina y alcohol en pacientes deprimidos. Litio: Se recomienda que se controlen los niveles plasmáticos de fítio al comienzz del tratamiente con Sertrina de torma que despues puedan hacerse los ajustes corres pondientes de la dosis de litio en caso necesario. La coadministración con litio puede conducir a una mayor incidencia de electos secundarios asociados con la serotonina (5-hT). Farmacos serotoninergicos. Lo duracion del pentodo de lavado que debe existir antes dol cambio desde un ISRS a otro no ha sido establecida. Debe realizarse un juicio clínico cuidadoso y prudente, particularmente cuando el cambio se realice desde fármacos de acción prolongada. Hasta que se disponga de más datos, los tármacos serotoninérgicos como triptoffano o fenfluramina, no deben usarse de forma concomitanse con Sertralina. Tratamiento electroconvulsivante (TEC): No existen estudios clínicos que establezcan los riesgos o beneficios del uso combinado de TEC y Sertralina. Inducción de las enzimas microsomales: Sertralina no presenta un efecto inductor clínicamente significativo sabre las enzimas hepá. ticas. Hipoglucemiantes: La coadministración de Sertralina con tolbutamida dio lugar a pequeños cambios estadisticamente significativos de algunos parámetros farmacocinéticos. No se ha observado interacción con glibenclamida. Estudios en animales han demostrado una interacción potencial con insulina, desconociéndose el significado clínico de este hecho hasta el momento. Otras interacciones: Puesto que Senralina se une a las proteinas plasmáticas, debe tenerse en cuenta el potencial de Sertralina para interaccionar con otros tármacos unidos a las proteinas plasmáticas. La coadministración de Sertralina y diazepam dio lugar a pequeños cambios, estadísticamente significativos de algunos parámetros far: macocinéticos. La administración conjunta con cimetidina provocó una disminución susmacocineticos. La mestistracion conjunta con cima lina provoct una disminución sustancial del aclaramiento de Sertralina. Se desconoce la significación clínica de estos hallazgos. Sertralina no tene efecto sobre la acción beta-bloqueante del atenolal. No se ha observado interacción con digoxina. Su coadministración con warfarina desencadenó un pequeno incremento aunque estadisticamente significativo del tiempo de protrombina, desconociéndose el signilcado clico de este efecto. Por consiguiente, debe controlarse cuidadosamene ch the Sertralina. Efectos secundarios: Los efectos secundarios que han aparecido con una trecuencia significativamente mayor que con placebo fueron: náuseas, diarrea/heces blandas, dispepsia, temblor, vertigos, insomnio, somnolencia, incremento de la sudoración, sequeded de boca y distunción sexual masculina (principalmente eyaculación retardada). Con muy poca trecuencia $\{0,8 \%\}$ se han comunicado elevaciones asintomáticas de las transaminasas séricas ( $\$ G O T$ y SGPT). Las alteraciones aparecieron normalmente en las primeras nueve semanas del tratamiento y desaparecieron rápidamente tras la interrupción del mismo. Excepcionaimente se han comunicado casos de hiponatremia que fueron reversibles tras la interrupción del tratamiento con Sertralina. A diferencia de los antidepresivos triciclicos, no se ha observado aumento de peso con el tratamiento; por el contrario, algunos pacientes tratados con Serttalina pueden experimentar reducción del peso corporal. No se ha observado que Sertralina produzca dependencia fisica o psiquica. Sobredosificación: BESITRAN(Sertralina) tiene un amplio margen de seguridad en el caso de sobredosificación. No se han comunicado secuelas graves después de una sobre. dosis única de Sertralina de hasta $6 \mathrm{~g}$. Aunque no se han comunicado muertes cuando sertralina fue administrada sola, sí se han comunicado fallecimientos en casos en que se tomaron sobredosis de Sertralina en asociación con otros fármacos yo alcohol Por consiguiente, todos los cosos de sobredosis deben ser tratados de forma agresiva. No se reco. mienda tramiento especifico y no existen antidotos especificos para Sertra ina Se debe establecer y mantener la via aérea, asegurar una oxigenación adecuada y ventilación. El establecer y mantener la via aerea, asegurar una oxigenación adectada y ventilación. El carbón activàdo, que puede utilizarse con sorbital, puede ser tanto o más eficaz que la emesis o lavado gástrico, y debe considerarse como tratamiento de la sobredosis. Se secomienda el control cardiaco y de los signos vitales junto con medidas generales de soporte y sintomateas. Debido al gran volumen de distribucion de Sertralina, es improbable que la diuresis forzada, dialisis, hemoperfusión y la plasmalêresis sean beneficiosas. Lista de excipientes: Cada comprimido contiene como excipiente. fosfato cácico dibásico, celulusa microcristalina, hidroxipropil celulosa, almidón, glicolato sódico, estearato magnésico, hidroxipropil metil celulosa, polietilen glicol, polisorbatos, díxido de titanio (E177). Incompatibilidades: No se han descrito. Caducidad: Cuatro años. Precauciones especiales de conservación: Lugar seco y temperatura inferior a $30^{\circ} \mathrm{C}$. Presentaciones y PVP (NA): BESITRAND $50 \mathrm{mg}$, envase con 30 comprimidos, 5.799 ptas.,IVA) BESITRAN $100 \mathrm{mg}$, envase con 30 comprimidos, 8.698 ptas (NA). Con receta médica. Especialidad reembolsable por los Organismos de la Seguridad Social. Aportación reducida, Instrucciones de uso/manipulación: No son necesarias instrucciones especiales. Consulte la ficha técnica completa del producto antes de prescribir. Para más información, dirigirse a: Laboratorios PFIZER, S.A. Principe de Vergara, 10928002 MAORID. 
Más Ventajas, Menos Complicaciones

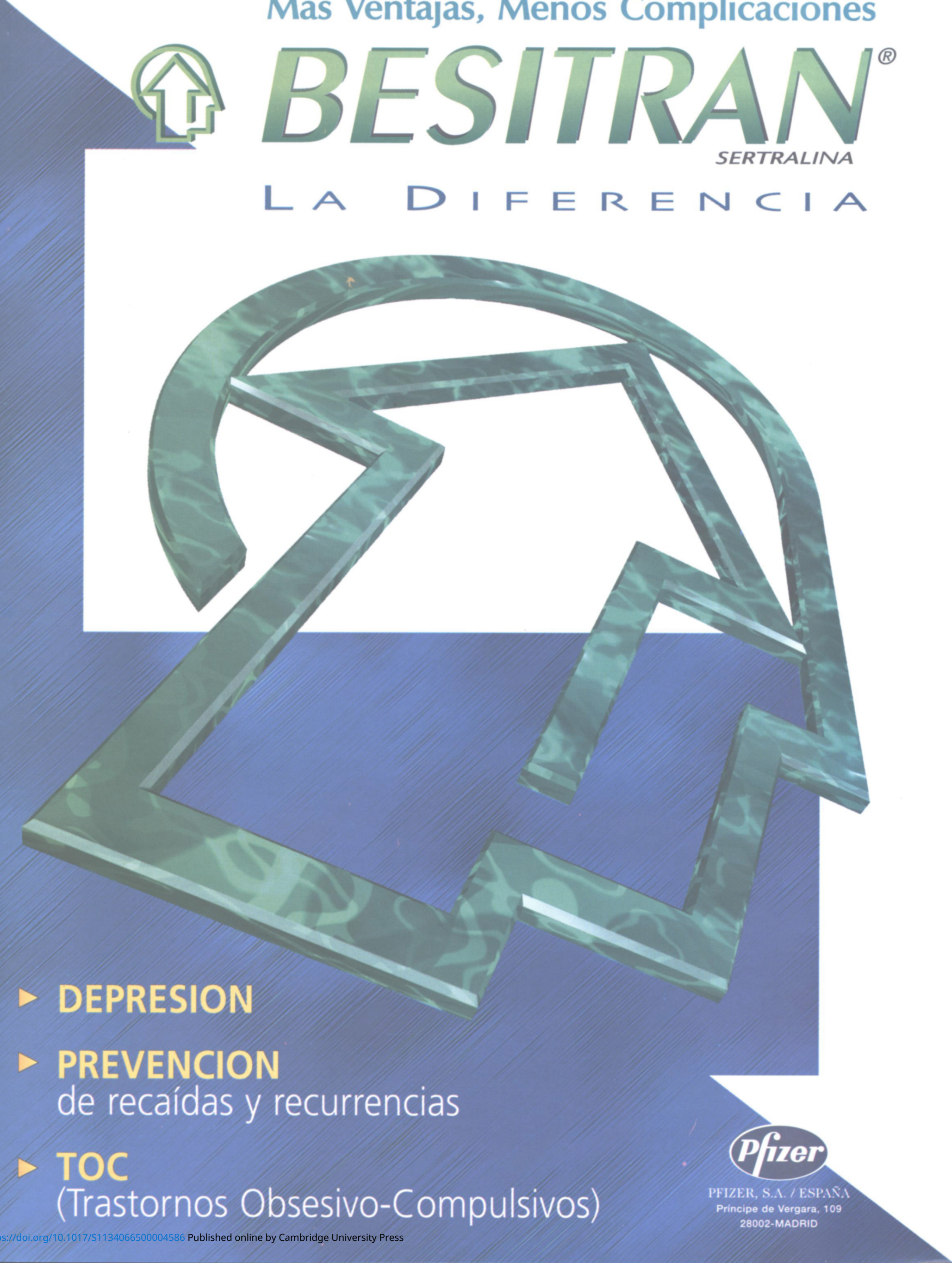




\section{EUROPEAN PSYCHIATRY.}

\author{
EDICION ESPAÑOLA
}

\author{
Volume 4, Number 6, September 1997
}

REVIEW ARTICLE

Short-term safety profile of zolpidem: objective measures of cognitive effects.

A. C. DeClerk, J. C. Bisserbe

\section{ORIGINAL ARTICLES}

Inter-rater reliability of ICD-10 in Chinese patients.

A. H. T. Pang, G. S. Ungvari, C. K. Wong, T. Leung...........

Are there differential effects of fluoxetine in retarded/blunted affect versus agitated/anxious depressives?

A. Partiot, R. Jouvent, A. Pierson, P. Baruch, J. N. Beuzen, S. Ammar, D. Widlöcher.

Cortisol-binding globulin levels in bipolar disorder.

E. Vieta, C. Gastó, M. J. Martínez de Osaba, A. Otero, E. Nieto, L. Pintor, J. Blanch, J. Vallejo...

Initial response to active drug and placebo predicts outcome of antidepressant treatment.

S. Priebe, M. Bröker

Attributional style and delusions: an investigation based on delusional content.

H. M. Sharp, C. F. Fear, D. Healy

\section{SHORT COMMUNICATION}

Sleep panic attacks in patients with panic disorder: the association with major depression.

M. Y. Agargün, H. Kara...

\section{AEP NEWS}

The European launch of interpersonal psychoterapy in the Xth World Congress of Psychiatry.

J. Solé-Puig
RENEURON 20 mo (FIuoxetina DCI). COMPOSICION: RENEURON 20 mg cásulas:Cada cápsula contiene fluoxetina

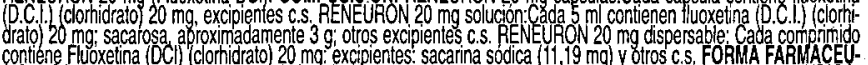

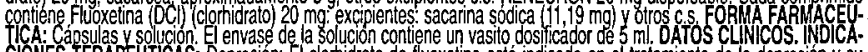
CIONES TERAPEUTICAS: Depresion: El Clontidrato de fluoxetina está indicado en el tratamiento de la depresión y su ansiedad asociada (DSM III, ICD-9 Y ROC). La eficacia de fuoxetina se establecio en ensayos cinicos de 5 a 6 semanas

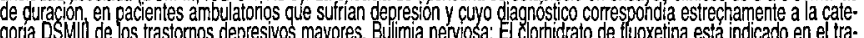

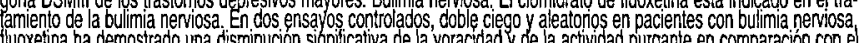
acho

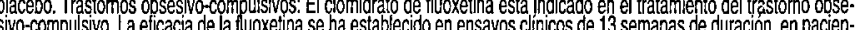

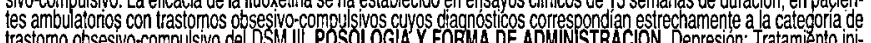
trastomo obsesivo-compulsivo del DSM III. POSOLOGIA Y FORMA DE ADMMN STRACION. Depresión: Tratamiento inicial: La dosis incial recomendada es de 20 mg al cia por la manana. Despues de varas semaras de tratamientio, yen deben adminisistrarse dos veces a cla (por ejemplo, por l a manana y al mediodia) y no debe excederse la cosis maxima de 80 myda. Como sucede con ortos antidepresivos, para que se alcance el electo antidepresvo tola, puede necesilarse un tempo de 4 o mas semanas de tratamiento. Mantenimiento, continuacion, tratamiento prolongado: No existen datos suficientes para poder hacer una recomendación en cuanto al tiempo que debe mantenerse el trazamientio de las personast tratadas con fluoxetina. En general, los episodios agudos de depresion necesitan varios meses de farmacoterapla sosmia nerviosa: La dosis recomendada es de $60 \mathrm{mg}$ al dia. Trastomos obsesivo-compulsivos: La dosis de $20 \mathrm{mod}$ dia. a 60

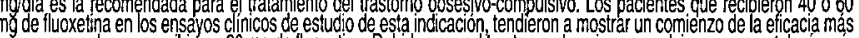
temprana que los que recibieron $20 \mathrm{mg}$ de fluoxetina. Debido a que el trastomp obsesiviv-compulsivo es una pato logia cro:

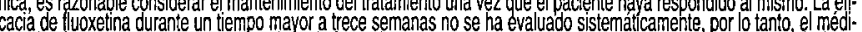

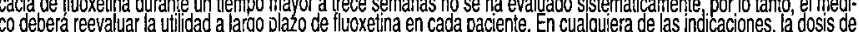

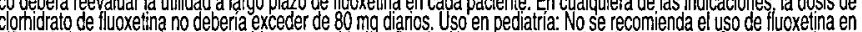
inos, dado gue e 0 se han establecido su sequidida Y eficacia. Uso en pacientes de edad avanzada: Se recomenda una dosis dlaria de 20 mg. Insúficiencia hepática: Se deben utílizar dosis menores o menos frecuentes. Insurficiencia renal: En

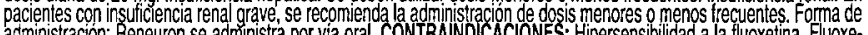
administracion: Reneuron se admínistra DOr via oral. CONTRAN DICACIONES: Hipersensibilidad a la fluoxetina. Fluoxetina no debe administrarse en combinacion con un innibicor de la moncaminooxidasa (IMAO), ni tampoco durante los 14 dias posteriores a a suspension del tratamientio con un

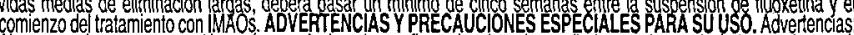
Alggunos pacientes con erupción cutánea, relacionada con fluoxetina, han desarrolladeo reacciones s sistemicas serias, posi. alerico ne azicar (sacarosal en su composicion 3 por cada $5 \mathrm{ml}$ aproximadamente, por' lo que tendrán que tenerlo en cuenta as personas diabeiticas". Precauciones: Se bebe llevar a cabo una estrecha montorzacion de los pacientes a comienzo del ratamianiento ya que la posibilidad de un intento de suicidio es inherente a la deoressón y puede persistir hasta que se

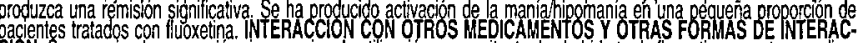
CION: Se recomienda precaucion $s$ s se requilere la utillzacion concomiante de clomidrato de fluoxetina con orros medicamentos activos a nive des sistema reevioso cental, ncluyendo el itito. Puede haber tanto aumento como cisminuclion de

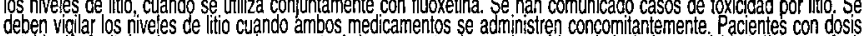
deben vicilar los nivees de ltitio cyando ambos medicamentos se administren concomitariemente. Pacientes con dosis

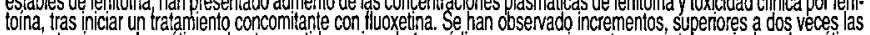
concentraciones plasmálicas de otros antidepresivos hetererciclicicos, que previamentie presentiaban niveles plasmáticos tos ou, son moto

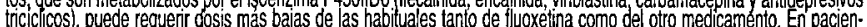

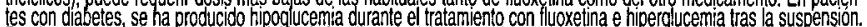

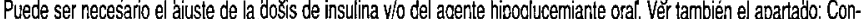

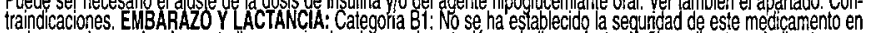
mujeres embarazadas. Los estudios en animales de experimentación no incican éectos danininos directos o indirectos respecto al desarrollo del embrion oleto la gestacion y el cesarrollo per y posthatala. Fuoxétina no debe utilizarse en pacien-

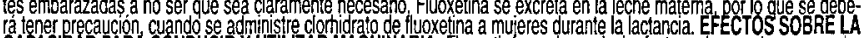

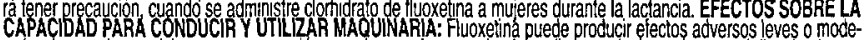
rados. Los pacienties deben tener precaucion cuando manejen maquinaras pueliorosas, incluyendo automonoviles hasta oue

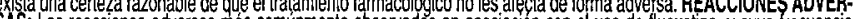
SAS: Las reacciones adversas mas comunmente observadas en asociacion con el uso de fluoxetina, y cuyja frecuencia encl

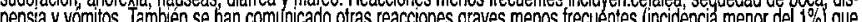
incluyen: sincope, arritmia cardiaca, anomallias en las. pruebas de tunción hepatica, aumento del tiempo de hemorracia, sindrome cerefral acudo y convusiones. ver tambien apartado:Advertencias y precauciones especiales de empleo. tes en la sobrecosis de fluoxetina. Otios sintomass pueden ser. agitación, convulsiones, inquietud, hipomaniá y otros sionos de excitacion de sistema nervioso central. Desde su comercialización, las comunicaciones dé casos de muerre ant. buidas a una sobrecossis de fluoxetina sola han sido extremadamente raras. Hasta diciembre de 1987, se habian comuncado dos muertes entre 38 comunicaciones de sobredossis aguda con fluoxetina, tanto sola como en combinación con otros medicamentos yo accoho. Una de as mueries ocurrio en un paciente que tomo una sobredoss de $1800 \mathrm{mg}$ de lluoxetina en combinacion con una cantidad no deteminada de maprotilina. Las concentraciones plasmmílicas de fluoxetina y

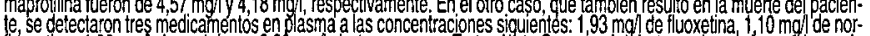

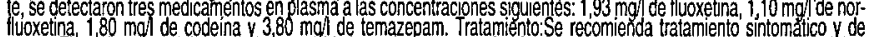

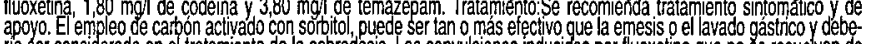
ria ser considerado en el iratamiento de la sobredosis. Las convulsiones inducidas por fluoxetina que no se resulvan de marera espontánea pueden responder a diazepam. En el manejo de la sobredosis se debe considerar la posibilidad de que el paciente haya ingerido una diversidad de fármacos. En pacientes que esten tomando tluoxetina o recientemente la hayan tomado e ingieran una cantidad excesiva de anitidepresivos se deberia proceder con especial cuidado, ya que en tales casos se podifia aumentar la posibilidad de secuelas clinicamentes significativas y yumentar el tiempo reguedido de CAS: Fuoxetina es un mecicamento de accion antidepresiva cuyo mecanismo de acción parece estar relaciona do conta NETICAS: Desoves de 6 a 8 \& horas de e una dosis oral unicica se observan concentraciones pico plasmaticas de fluoxetina. os alimentos no parece oue atecten la biodisponibilicad sistemica de thuoxelina. Fluoxetina se oistribulye ampllamentie por el cuerpo uniendose muchio a proteinas plasmáticas. La vida media de eliminacion de tuloxetina es de 46 dias siendol a

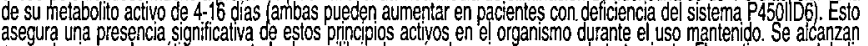
asegura una presencia significaliva de estos priccpios actios en el organismo durante el uso manterido. Se acanzant

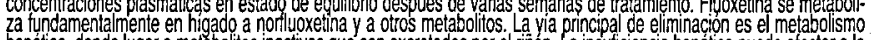

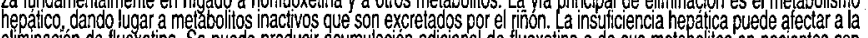
eiminación de fluoxetina Se puede producir acumulacion adicional de fluxetetina o de sus metabolitios en pacientes con

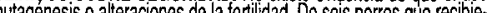

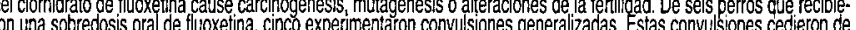

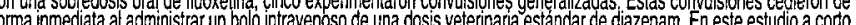
plazo, a concentración más baja de fluoxetina con la culal se presentaror convulsiones, fue tan solo el doble de la con-

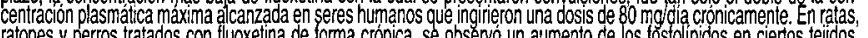

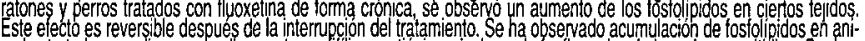
males tratados con diversos medicamentos anifilicicos catiónicos, incluyendo fenfluaramina imipramina y ranitidina Se desGENEURON $20 \mathrm{mg}$ capsulas:Almidion y silicona atuida 350 csil. RENEURON $20 \mathrm{mg}$ solucion: Sacarosa, acido benzoico, icerina. saborizante de menta. REN EURON $20 \mathrm{mg}$ dispersable: sacarina sodica, célulosa microcristalina, manitol, sorbiTol, aroma de anis, aroma de peppemint cioxido de silcio colodal, almidon fluente seco, tumarato sodico de esteanilo y

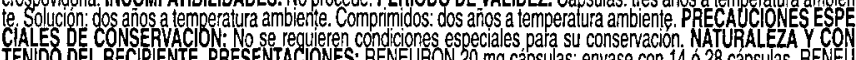

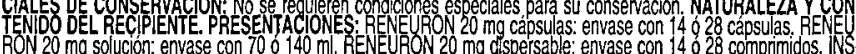

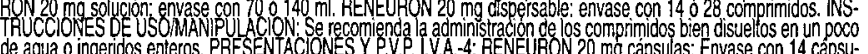

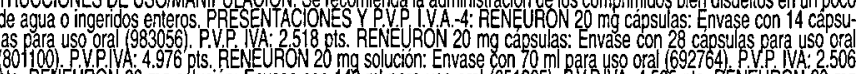

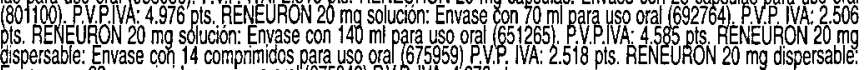

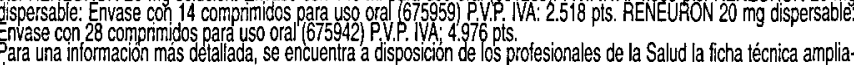
Pará una información mas delallada, se encuentra a disposición de los profesionales de la Salud la ficha técrica amplia- 


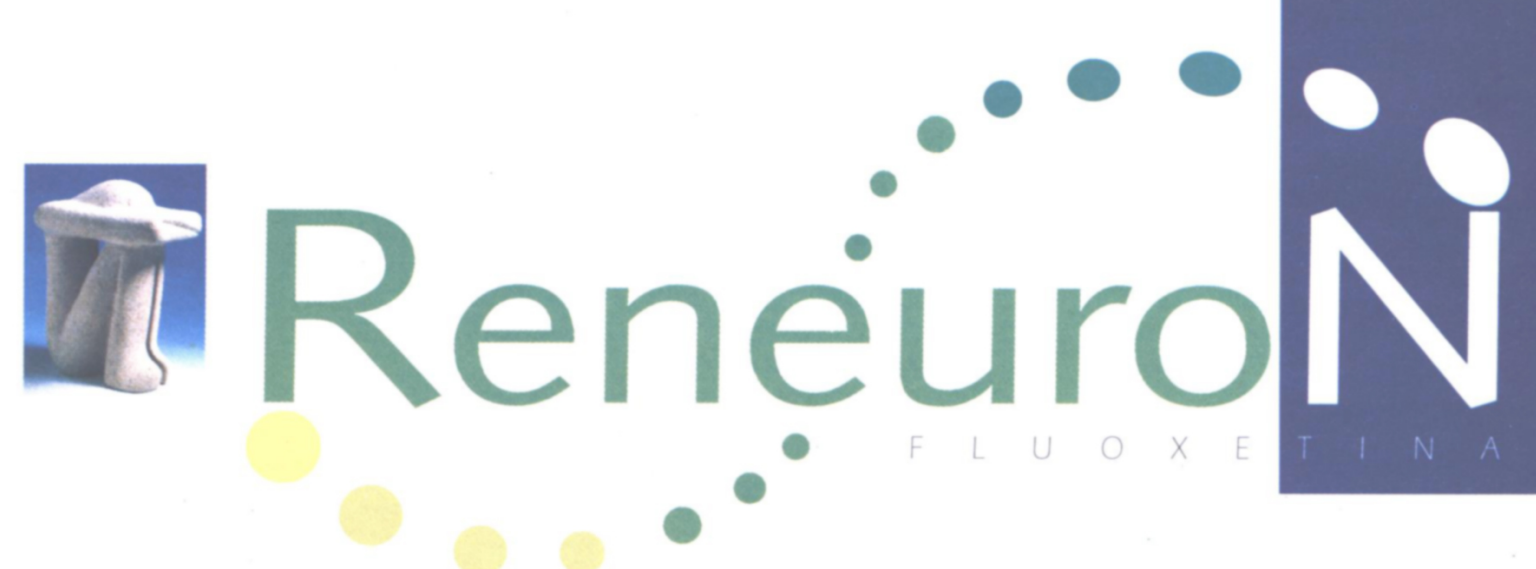

\section{Unico ISRS con indicación de Depresión y su Ansiedad asociada}

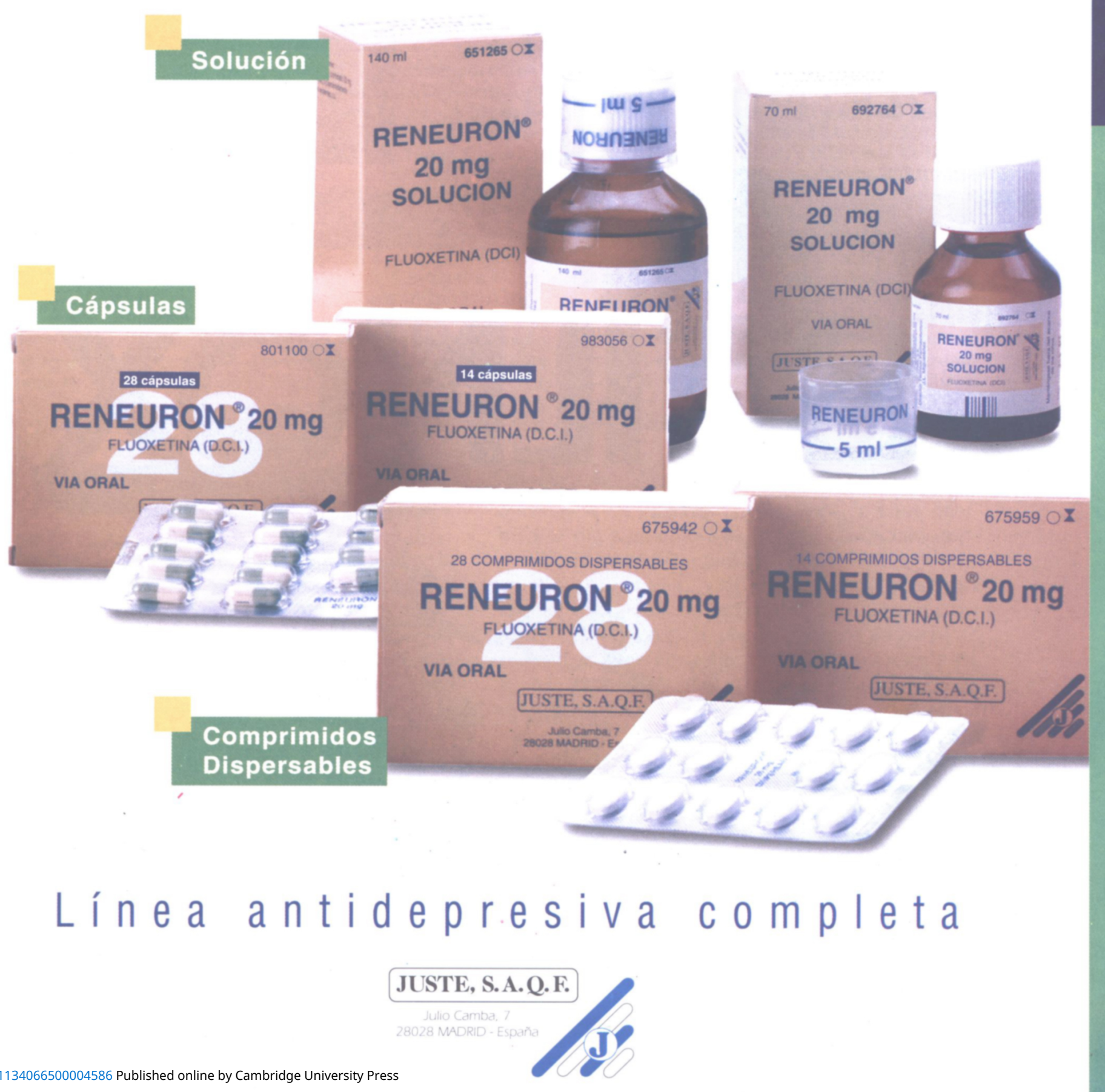




\section{medicus mundi \\ ÚNETE A NOSOTROS POR UN MUNDO MÁS SOLIDARIO}

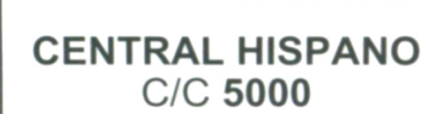

C/C 5000

\section{TLF.: (91) 3195849}

medicus mundi agradece la inserción gratuita de este anuncio

ARGENTARIA

C/C 30-50000-D

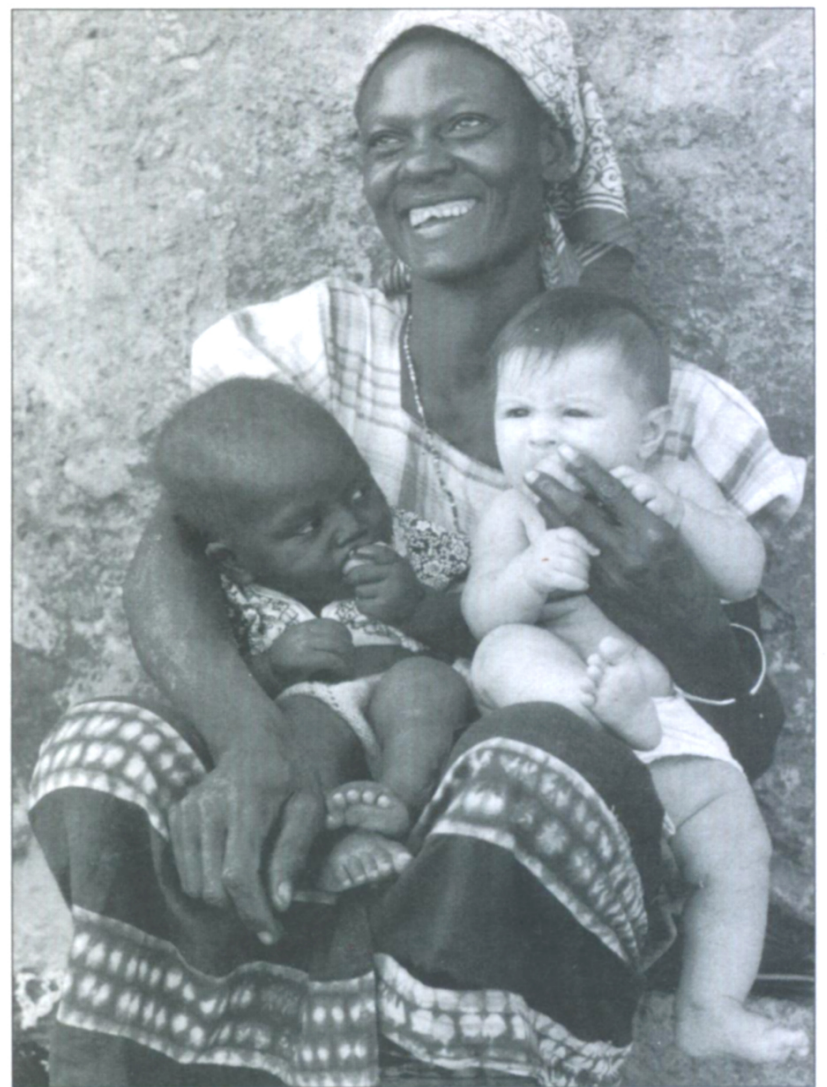

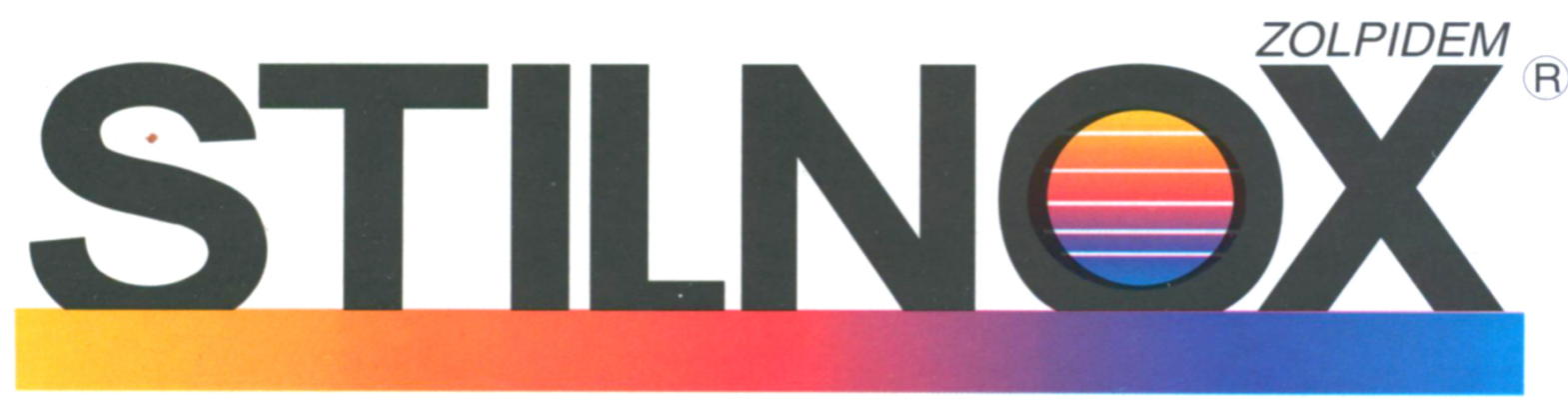

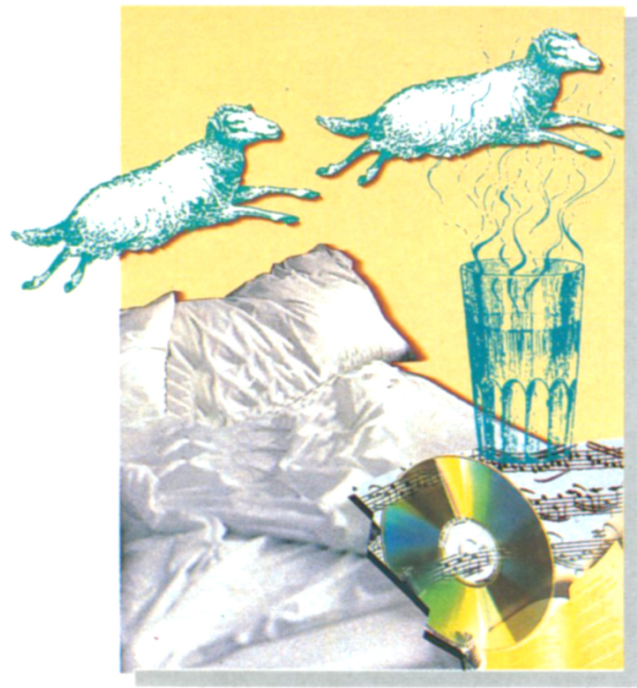

